\title{
Low-Dose Dual-Energy Computed Tomography for PET Attenuation Correction with Statistical Sinogram Restoration
}

\author{
Joonki Noh ${ }^{\dagger}$, Jeffrey A. Fessler ${ }^{\dagger}$, Paul E. Kinahan ${ }^{\ddagger}$ \\ $\dagger^{\dagger}$ Department of Electrical Engin. and Computer Science, Univ. of Michigan, Ann Arbor, MI, \\ ${ }^{\ddagger}$ Department of Radiology, Imaging Research Lab., Univ. of Washington, Seattle, WA, USA.
}

\begin{abstract}
Dual-energy (DE) X-ray computed tomography (CT) has been proposed as an useful tool in various applications. One promising application is DECT with low radiation doses used for attenuation correction in positron emission tomography (PET). In low-dose DECT, conventional methods for sinogram decomposition have been based on logarithmic transformations and ignored noise properties, leading to very noisy component sinogram estimates. In this paper, we propose two novel sinogram restoration methods that are statistically motivated; penalized weighted least square (PWLS) and penalized likelihood (PL), producing less noisy component sinogram estimates for low-dose DECT than the conventional approaches. The restored component sinograms can improve attenuation correction, thus allowing better image quality in PET. Experiments with a digital phantom indicate that the proposed methods produce less noisy sinograms, reconstructed images, and attenuation correction factors (ACF) than the conventional one, showing promise for CT-based attenuation correction in emission tomography.
\end{abstract}

Keywords: PET/CT, dual-energy, attenuation correction, sinogram restoration

\section{INTRODUCTION}

Combing PET and X-ray CT systems in a single scanner has several benefits in medical diagnosis. Firstly, it provides the accurate alignment of functional and anatomical information obtained from the human organs. For example in oncology imaging, PET/CT can be used to improve the identification and localization of the lesions of the human organs such as cancers. Secondly, the CT images are used for the attenuation correction of the PET images, which is called CT-based attenuation correction (CTAC). The CTAC provides lower noise in ACFs and less scan time than the standard attenuation correction by PET transmission scan. ${ }^{1,2}$

In the CTAC for PET, CT images need to be transformed to estimates of the linear attenuation coefficients (LAC) evaluated at $511 \mathrm{keV}$. However, there is no exact way to transform LACs in the range of CT energies $(30 \mathrm{keV} \sim 150 \mathrm{keV})$ to LACs at the PET energy $(511 \mathrm{keV})$. Some methods for this conversion have been proposed, e.g., segmentation or bilinear scaling for CT images obtained using a single-kVp source spectrum.

Compared to the conventional X-ray CT using a single-kVp spectrum, there are advantages in DECT, i.e., dual-kVp X-ray CT. For example, DECT requires no prior knowledge of object's attenuation properties for segmentation. It is thus possible to eliminate a potential source of errors by using DECT. ${ }^{3}$ Recent studies show that, when DECT scanners are integrated into PET scanners, it provides more accurate attenuation correction in the emission image reconstruction by reducing bias compared to single-kVp $\mathrm{CT}$, therefore improving PET images. $^{4,5}$ When the main purpose of DECT is the attenuation correction in PET images, one should use low $\mathrm{X}$-ray doses, requiring statistical methods for sinogram restoration or image reconstruction to minimize noise. Increased scan time by DECT can be avoided by alternating the incident spectra between projection angles or slices or conceivably in other arrangements.

We can make ACFs for PET in the sinogram domain without having to reconstruct DECT images. Therefore, with a polyenergetic measurement model to correct beam-hardening artifacts, we mainly focus on developing iterative algorithms for sinogram restoration in DECT based on proper statistical models in this paper. Since the

Further author information: (Send correspondence to Joonki Noh)

Joonki Noh: E-mail: nohjoonk@umich.edu,

Jeffrey A. Fessler: E-mail: fessler@eecs.umich.edu,

Paul E. Kinahan: E-mail: kinahan@u.washington.edu.

Medical Imaging 2008: Physics of Medical Imaging, edited by Jiang Hsieh, Ehsan Samei, Proc. of SPIE Vol. 6913, 691312, (2008) · 1605-7422/08/\$18 · doi: 10.1117/12.769855

Proc. of SPIE Vol. 6913 691312-1 
spatial resolution of usual PET images is modest, the computational cost of sinogram restoration in DECT can be practical. Sinogram restoration approaches based on appropriate statistical models can perform well even at low doses, whereas the conventional sinogram decomposition performs poorly in low signal to noise ratio (SNR). The proposed DECT methods generalize previous restoration approaches for single-kVp CT. ${ }^{6}$

This article is organized as follows. We start with the discussion of a polychromatic measurement model and a basic material decomposition for DECT in section 2. For sinogram restoration in DECT, we first review the conventional sinogram decomposition method in section 3.1. Then, two statistically motivated approaches, PWLS and PL are introduced and their iterative algorithms are derived by the optimization transfer principle in section 3.2 and section 4 . Some simulation results to compare the conventional approach and proposed methods are provided in section 5 . Finally, the conclusions are drawn in section 6 .

\section{POLYCHROMATIC MEASUREMENT MODEL}

We consider a general measurement model based on polychromatic source spectra for multiple-kVp CT in this section. Transmission tomographic measurements are collected for $M_{0}(\geq 1)$ different incident spectra. For each incident spectrum, forward projections (line integrals) are recorded for $N_{d}$ radius-angle pairs, forming a component sinogram. We let $y_{m i}$ denote the measurement for the $m$ th incident spectrum and the $i$ th ray, where $m=1, \ldots, M_{0}$ and $i=1, \ldots, N_{d}$. We assume that $y_{m i}$ is a random variable whose ensemble mean $\bar{y}_{m i}$ is defined by an underlying physical phenomenon as

$$
\mathbf{E}\left[y_{m i}\right]=\bar{y}_{m i} \triangleq \int I_{m}(\mathcal{E}) \exp \left(-\int_{\mathcal{L}_{i}} \mu(\vec{x}, \mathcal{E}) \mathrm{d} \ell\right) \mathrm{d} \mathcal{E}+r_{m i},
$$

where $I_{m}(\mathcal{E})$ denotes the product of the $m$ th source spectrum and the detector gain, and $\int_{\mathcal{L}_{i}} \cdot \mathrm{d} \ell$ is the line integral along the $i$ th ray. $\mu(\vec{x}, \mathcal{E})$ represents the LAC of the scanned object at the spatial location $\vec{x}$ and the energy level $\mathcal{E}$, and $r_{m i}$ denotes known additive background contributions such as room background, dark current and scatter. By considering the polychromatic nature of $M_{0} \mathrm{X}$-ray sources spectra, i.e., the integral over the energy in (1), correction for beam hardening effects can be made automatically in the algorithms for sinogram restoration that will be developed in section 3.2 and section 4 .

Since the measurements $y_{m i}$ are finite whereas $\mu(\vec{x}, \mathcal{E})$ is a continuous function of the location $\vec{x}$ and the energy $\mathcal{E}$, we need to parameterize the LAC. We model $\mu(\vec{x}, \mathcal{E})$ using a set of basis functions that are separable in the space and energy domain as follows: ${ }^{7}$

$$
\mu(\vec{x}, \mathcal{E})=\sum_{l=1}^{L_{0}} \beta_{l}(\mathcal{E}) \rho_{l}(\vec{x}),
$$

where $\beta_{l}(\mathcal{E})$ denotes the energy-dependent mass attenuation coefficient (MAC) and $\rho_{l}(\vec{x})$ is the density map of the $l$ th material type for $l=1, \ldots, L_{0}$. $L_{0}$ thus denotes the number of material types comprising the object. Usually, $M_{0}=2$ and $L_{0}=2$, e.g., soft tissue and bone, are set in DECT. Other material decompositions are possible. $^{8}$

Substituting (2) into (1) and simplifying yield the following expression for the ensemble mean $\bar{y}_{m i}$ :

$$
\begin{gathered}
\bar{y}_{m i}=I_{m} e^{-f_{i m}\left(\boldsymbol{s}_{i}\right)}+r_{m i}, \\
f_{i m}\left(\boldsymbol{s}_{i}\right) \triangleq-\log v_{i m}\left(\boldsymbol{s}_{i}\right), \quad v_{i m}\left(\boldsymbol{s}_{i}\right) \triangleq \int \frac{I_{m}(\mathcal{E})}{I_{m}} e^{-\sum_{l=1}^{L_{0}} \beta_{l}(\mathcal{E}) s_{l i}} \mathrm{~d} \mathcal{E},
\end{gathered}
$$

where the nonlinear function $f_{i m}\left(s_{i}\right)$ characterizes the beam hardening caused by polychromatic X-ray source spectra. We define the total intensity $I_{m}$ for the $m$ th incident spectrum and sinogram vector $s_{i}$ at the $i$ th ray as

$$
I_{m} \triangleq \int I_{m}(\mathcal{E}) \mathrm{d} \mathcal{E}, \quad s_{i} \triangleq\left(s_{1 i}, \ldots, s_{L_{0} i}\right)^{T}, \quad s_{l i} \triangleq \int_{\mathcal{L}_{i}} \rho_{l}(\vec{x}) \mathrm{d} \ell .
$$

It can be shown that the nonlinear function $f_{i m}\left(s_{i}\right)$ has the following two properties. For any $(l, i, m)$, we have

$$
\frac{\partial f_{i m}\left(\boldsymbol{s}_{i}\right)}{\partial s_{l i}}>0, \quad-\nabla^{2} f_{i m}\left(\boldsymbol{s}_{i}\right) \succeq 0,
$$


where the inequality " $\succeq "$ is in the matrix sense. Therefore $f_{i m}\left(\boldsymbol{s}_{i}\right)$ is a strictly monotonic increasing and concave function with respect to $s_{i}$. These properties are useful to determine the curvatures of the surrogate functions for PWLS in section 3.2.

For convenient numerical implementation, there have been several methods to approximate $f_{i m}\left(\boldsymbol{s}_{i}\right)$. Instead of the conventional approach of using polynomial approximations, we exploit an exponential approximation that imitates the definition of $f_{i m}$ in (4). We assume

$$
f_{i m}\left(\boldsymbol{s}_{i}\right) \approx \tilde{f}_{i m}\left(\boldsymbol{s}_{i}\right) \triangleq-\log \left(\sum_{k} \alpha_{i m k} e^{-\beta_{i m k}^{T} \boldsymbol{s}_{i}}\right),
$$

where we require $\alpha_{i m k} \geq 0$ and $\sum_{k} \alpha_{i m k}=1$ because we can treat $I_{m}(\mathcal{E}) / I_{m}$ as a probability density function with respect to $\mathcal{E}$. The coefficients $\alpha_{i m k}$ and $\beta_{i m k}$ can be determined by non-negative least square (NNLS). After fitting (7) to the sample values of $f_{i m}\left(s_{i}\right)$ evaluated at the lattice locations of $\boldsymbol{s}_{i}$ inside an appropriate rectangular region, we obtain approximations to the first derivative and second derivative of $f_{i m}$ at the other locations of $s_{i}$. These approximations are useful for implementing the developed algorithms by PWLS and PL in the following sections.

\section{LOGARITHMIC TRANSFORMATION BASED METHODS}

Before proposing a statistical method based on PL, two approaches that are based on a logarithmic transformation of the measurements are described in this section. The first method is the conventional non-statistical sinogram decomposition and the second one is a statistically motivated sinogram decomposition by PWLS.

\subsection{Conventional Sinogram Decomposition}

For a given noisy measurement $y_{m i}$, an easy and naive method to estimate $f_{i m}\left(\boldsymbol{s}_{i}\right)$ is to invert (3). By ignoring measurement noise and inverting (3), we have the following estimate of $f_{i m}$ :

$$
\widehat{f}_{i m} \triangleq-\log \left\{\operatorname{smooth}\left(\frac{y_{m i}-r_{m i}}{I_{m}}\right)\right\}
$$

where radial smoothing is often applied to reduce noise. ${ }^{9}$ By equating $\widehat{f}_{i m}$ to $f_{i m}\left(\boldsymbol{s}_{i}\right)$ in $(4)$, we have a system of $M_{0}$ nonlinear equations with $L_{0}$ unknowns for the $i$ th ray. Since it is usual to choose $M_{0}=L_{0}$, simply solving this system of equations yields an estimate of the sinogram vector of the following form:

$$
\widehat{\boldsymbol{s}}_{i} \triangleq \boldsymbol{f}_{i}^{-1}\left(\widehat{\boldsymbol{f}}_{i}\right)
$$

where $\boldsymbol{f}_{i} \triangleq\left(f_{i 1}, \ldots, f_{i M_{0}}\right)^{T}$ for $i=1, \ldots, L_{0}$. This is called the classical sinogram decomposition, and it involves a noise amplifying step. After estimating $\boldsymbol{s}_{i}$ for all $i$, the next step is usually to use these component sinograms to reconstruct component images, e.g., soft tissue and bone images, by separately applying filter back projection (FBP). This procedure produces unacceptably noisy estimates of the component images, limiting the use of the reconstructed images for attenuation correction of PET images.

\subsection{Sinogram Decomposition with PWLS}

Instead of solving the nonlinear equations with the estimates in (8), we propose to estimate component sinograms by a statistically motivated method: minimizing a PWLS cost function. Such a method requires an iterative algorithm to restore component sinograms, thus computationally more expensive than the conventional sinogram decomposition. However, iterative methods usually provide much less noisy sinogram estimates and the overall computational cost, when the PWLS method is combined with FBP, is not as expensive as full iterative methods for the reconstruction of CT images. Using $\widehat{f}_{i m}$ from (8), we estimate the component sinograms by conducting the following PWLS minimization. Subject to the non-negativity of the elements of $\mathbf{s}$, we have

$$
\widehat{\mathbf{s}}_{P W L S}=\underset{\mathbf{s} \in \mathbf{R}^{L_{0} \times N_{d} \geq 0}}{\arg \min } \Phi(\mathbf{s}) \triangleq \underset{\mathbf{s} \in \mathbf{R}^{L_{0} \times N_{d} \geq 0}}{\arg \min } \sum_{i=1}^{N_{d}} \frac{1}{2}\left(\widehat{\boldsymbol{f}}_{i}-\boldsymbol{f}_{i}\left(\boldsymbol{s}_{i}\right)\right)^{T} \boldsymbol{D}_{i}\left(\widehat{\boldsymbol{f}}_{i}-\boldsymbol{f}_{i}\left(\boldsymbol{s}_{i}\right)\right)+R(\mathbf{s}),
$$


where the sinogram matrix whose size is $L_{0} \times N_{d}$ is defined as $\mathbf{s} \triangleq\left\{\boldsymbol{s}_{i}\right\}_{i=1}^{N_{d}}$ and the weight matrix whose length is $M_{0} \times M_{0}$ is given by $\boldsymbol{D}_{i} \triangleq \operatorname{diag}_{m=1}^{M_{0}}\left\{y_{m i}\right\}$ for $i=1, \ldots, N_{d}$. If the measurements are approximately Poisson distributed and $r_{m i}$ is small, this choice of $\boldsymbol{D}_{i}$ is reasonable based on the approximate variance ${ }^{10,11}$ of $\widehat{f}_{i m}$ given by

$$
\operatorname{var}\left(\widehat{f}_{i m}\right) \approx \frac{\operatorname{var}\left(y_{m i}\right)}{\left(\bar{y}_{m i}-r_{m i}\right)^{2}} \approx \frac{1}{y_{m i}} .
$$

The roughness penalty function $R(\mathbf{s})$ is generally expressed in the form of

$$
R(\mathbf{s}) \triangleq \sum_{l=1}^{L_{0}} \gamma_{l} \sum_{k=1}^{K} \psi_{l k}\left(\left[\mathbf{C}_{l} \check{s}_{l}\right]_{k}\right),
$$

where $\gamma_{l}$ is the regularization parameter that controls the tradeoff between the data fit and the roughness penatly for $l$ th material type, $\psi_{l k}$ is a convex potential function, $\mathbf{C}_{l}$ is a first-order difference matrix, and $\check{s}_{l}^{T}$ denotes a row vector of the sinogram matrix $\mathbf{s}$ associated with the $l$ th material type. In this paper, we use a quadratic penalty function, i.e., $\psi_{l k}(t) \triangleq t^{2} / 2$ and $\mathbf{C}_{l}=\mathbf{C}$ is chosen to regularize component sinograms only in the radial direction. So, $k \approx N_{d}$. We have the following penalty function:

$$
R(\mathbf{s})=\frac{\gamma_{1}}{2} \sum_{k=1}^{K}\left[\mathbf{C} \check{\boldsymbol{s}}_{1}\right]_{k}^{2}+\frac{\gamma_{2}}{2} \sum_{k=1}^{K}\left[\mathbf{C} \check{\boldsymbol{s}}_{2}\right]_{k}^{2} .
$$

We use the optimization transfer principle ${ }^{12}$ to perform the minimization in (10). Since the PWLS data fit term in (10) and $R(\mathbf{s})$ in (12) are additively separable, separable quadratic surrogates (SQS) can be easily derived in the framework of the optimization transfer principle and the non-negativity constraint on $\mathbf{s}$ can be imposed, allowing a simultaneous update of $s_{l i}$ for $l=1, \ldots, L_{0}$ and $i=1, \ldots, N_{d}$. We arrive at the following equation for the update of component sinogram estimates in the $n$th step:

$$
s_{l i}^{(n+1)}=\left[s_{l i}^{(n)}-\frac{1}{h_{l i}^{(n)}} \frac{\partial \Phi\left(s_{l i}^{(n)}\right)}{\partial s_{l i}}\right]_{+},
$$

where the subscript + is due to the non-negativity constraint on $s_{l i}$. The curvature $h_{l i}^{(n)}$ is defined by

$$
h_{l i}^{(n)} \triangleq \sum_{m=1}^{M_{0}} w_{i m}+\gamma_{l} \sum_{k=1}^{K} \frac{c_{k i}^{2}}{a_{k i}},
$$

where $c_{k i} \triangleq[\mathbf{C}]_{k i}$. If $c_{k i} \neq 0$, we set $a_{k i} \triangleq\left|c_{k i}\right| / \sum_{i^{\prime}=1}^{N_{d}}\left|c_{k i^{\prime}}\right|$. If $c_{k i}=0$, we set $a_{k i}=0$. The curvature piece from the WLS is given by

$$
w_{i m} \triangleq\left(\left\|b_{m}\right\|^{2}+\left[\widehat{f}_{i m}\right]_{+} \cdot\left\|-\nabla^{2} f_{i m}(0)\right\|\right) y_{m i}
$$

where $b_{m} \triangleq\left[\bar{\beta}_{1 m}, \ldots, \bar{\beta}_{L_{0} m}\right]^{T}$ and the effective MAC is given by

$$
\bar{\beta}_{l m} \triangleq \int \beta_{l}(\mathcal{E}) \frac{I_{m}(\mathcal{E})}{I_{m}} \mathrm{~d} \mathcal{E} .
$$

Note that the curvature $h_{l i}^{(n)}$ can be precomputed and the associated surrogate functions satisfy the surrogate conditions. Therefore, the updates provided by (14) decrease the PWLS cost function $\Phi(\mathbf{s})$. After estimating the component sinograms for $L_{0}$ material types, FBP can be separately applied to each sinogram $\left\{\widehat{s}_{i l}\right\}_{i=1}^{N_{d}}$ to reconstruct the component images if desired. However, we can obtain ACFs for PET images directly from the restored component sinograms by PWLS. 


\section{STATISTICAL SINOGRAM RESTORATION BY PENALIZED LIKELIHOOD}

The PWLS approach discussed in the previous section is statistically motivated. However, it requires the logarithmic transformation to obtain $\widehat{f}_{i m}$ in (8), so the solutions are suboptimal in terms of dose and noise. This section proposes a PL method based on a Poisson measurement model. By combining the Poisson noise model with the ensemble mean in (1), we assume the measurement $y_{m i}$ obeys a Poisson distribution, namely,

$$
y_{m i} \sim \operatorname{Poisson}\left(\bar{y}_{m i}\left(\boldsymbol{s}_{i}\right)\right) .
$$

This model leads to the following PL cost function:

$$
\Psi(\mathbf{s}) \triangleq-L(\mathbf{s})+R(\mathbf{s})=\sum_{m=1}^{M_{0}} \sum_{i=1}^{N_{d}} L_{m i}\left(\mathbf{s}_{i}\right)+R(\mathbf{s}),
$$

where the sinogram matrix is denoted as $\mathbf{s} \triangleq\left\{\boldsymbol{s}_{i}\right\}_{i=1}^{N_{d}}$ and the negative Poisson log-likelihood function for the $m$ th incident spectrum and $i$ th ray is defined by

$$
L_{m i}\left(\boldsymbol{s}_{i}\right) \triangleq \bar{y}_{m i}\left(\boldsymbol{s}_{i}\right)-y_{m i} \log \bar{y}_{m i}\left(\boldsymbol{s}_{i}\right),
$$

where $g_{m i}(x) \triangleq x-y_{m i} \log x$ is a convex function with respect to $x$. We use the same form of the roughness penalty function as (12) for the PL cost function.

We estimate the component sinograms by performing the following minimization:

$$
\widehat{\mathbf{s}}_{P L}=\underset{\mathbf{s} \in \mathbf{R}^{L_{0} \times N_{d} \geq 0}}{\arg \min } \Psi(\mathbf{s}) .
$$

We again use the optimization transfer principle ${ }^{12}$ to obtain a sequence of surrogates to compute $\widehat{\mathbf{s}}_{P L}$. Since both $\boldsymbol{L}(\mathbf{s})$ (data fitting term) and $R(\mathbf{s})$ (penalty term) are additively separable, SQSs can be easily derived in the framework of the optimization transfer principle and thus all estimates of $s_{l i}$ can be updated simultaneously for $l=1, \ldots, L_{0}$ and $i=1, \ldots, N_{d}$. One arrives at the following equation for the update of component sinogram estimates in the $n$th step. For given $l$ and $i$, we have

$$
s_{l i}^{(n+1)}=\left[s_{l i}^{(n)}-\frac{1}{d_{l i}^{(n)}} \frac{\partial \Psi\left(s_{l i}^{(n)}\right)}{\partial s_{l i}}\right]_{+},
$$

where the subscript + imposes the non-negativity constraint on the sinogram matrix s. The curvature $d_{l i}^{(n)}$ and effective energy of the $m$ th spectrum $\overline{\mathcal{E}}_{m}$ are defined as follows:

$$
d_{l i}^{(n)} \triangleq \sum_{m=1}^{M_{0}} \beta_{l}\left(\overline{\mathcal{E}}_{m}\right) \sum_{j=1}^{L_{0}} \beta_{j}\left(\overline{\mathcal{E}}_{m}\right) y_{m i}+\gamma_{l} \cdot \sum_{k=1}^{K} \frac{c_{k i}^{2}}{a_{k i}}, \quad \overline{\mathcal{E}}_{m} \triangleq \frac{\int \mathcal{E} I_{m}(\mathcal{E}) \mathrm{d} \mathcal{E}}{\int I_{m}(\mathcal{E}) \mathrm{d} \mathcal{E}},
$$

where $c_{k i} \triangleq[\mathbf{C}]_{k i}$ and we precompute the curvature $d_{l i}^{(n)}$ to accelerate the algorithm in (22). As in PWLS, if $c_{k i} \neq 0$, we set $a_{k i} \triangleq\left|c_{k i}\right| / \sum_{i^{\prime}=1}^{N_{d}}\left|c_{k i^{\prime}}\right|$, and if $c_{k i}=0$, we set $a_{k i}=0$. There are no easy way to show that the surrogate functions associated with the curvature $d_{l i}^{(n)}$ satisfy the surrogate conditions due to the approximations used to make $d_{l i}^{(n)}$ precomputable. Thus we may not have the monotonicity of the PL cost function $\Psi(\mathbf{s})$ with the updates provided by the algorithm in (22). However, if the approximations are reasonably accurate and $d_{l i}^{(n)}$ provides a sufficiently large curvature ensuring the surrogate conditions, we can expect the monotonicity when we implement (22). This can be checked empirically by investigating the value of $\Psi(\mathbf{s})$ in each iteration for given data. In fact, the simulations corresponding to the PL method exhibit monotonicity in section 5 . To reconstruct component images from $\widehat{\mathbf{s}}_{P L}$, FBP can be applied separately to each restored component sinogram as usual. However, we can make ACFs for PET directly from the sinogram domain. 

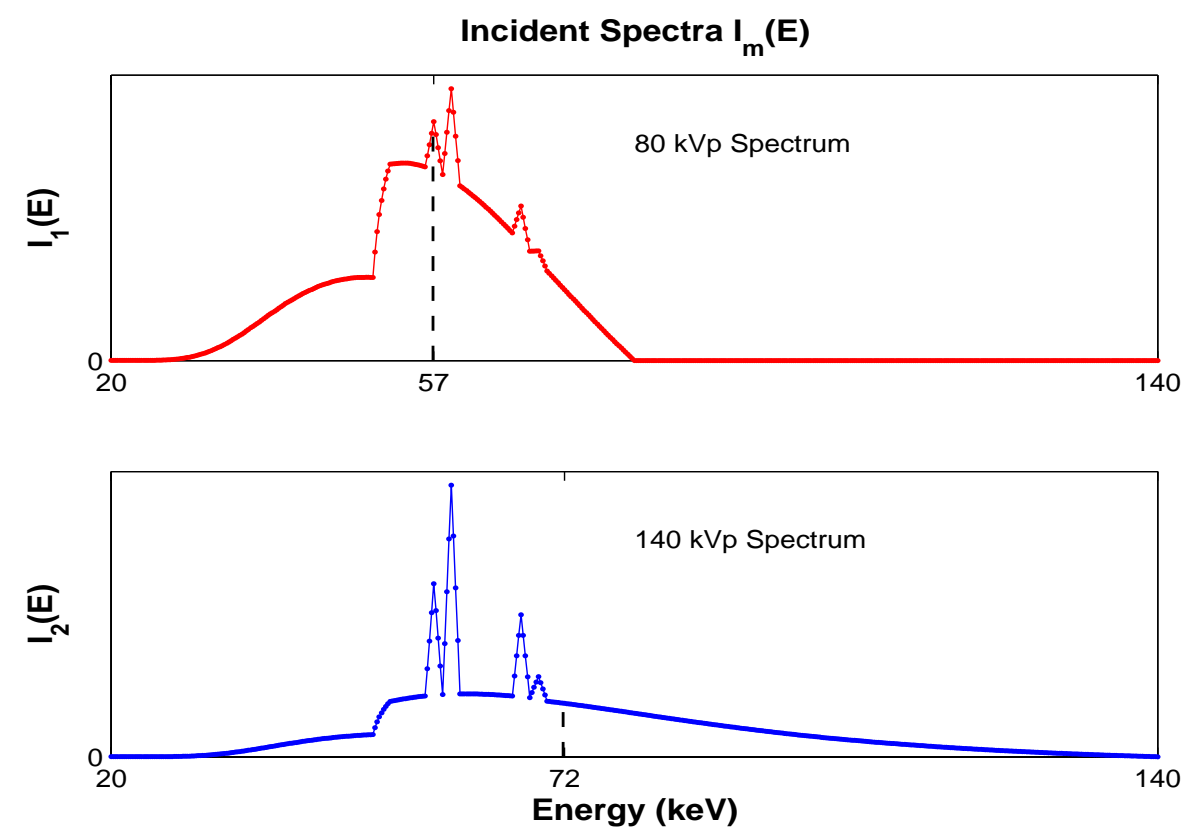

Figure 1. Two incident spectra $I_{m}(\mathcal{E})$ against $\mathcal{E}[\mathrm{keV}]$ for $m=1,2: 80 \mathrm{kVp}$ (top) and $140 \mathrm{kVp}$ (bottom). The dashed vertical line indicates the effective energy $\left(\overline{\mathcal{E}}_{m}\right)$ of each spectrum defined in $(23)$.

\section{SIMULATION EXPERIMENTS}

To compare the conventional sinogram decomposition and two proposed sinogram restoration methods, we performed simulations with a digital phantom consisting of soft tissue and bone. Two source spectra with $80 \mathrm{kVp}$ and $140 \mathrm{kVp}$ that are incident on the phantom are shown on Fig.1 with their effective energies $\left(\overline{\mathcal{E}}_{1}=57\right.$ and $\overline{\mathcal{E}}_{2}=72$ ). The true density maps of two material types are shown in the first row of Fig.3. From the true density maps, we synthesized the low-dose DECT measurements using Poisson random variables whose ensemble means are given by (1) for $m=1, \ldots, M_{0}$ and $i=1, \ldots, N_{d}$. To simulate low radiation doses, the number of incident photons per ray was set to $5 \times 10^{4}$ for the $140 \mathrm{kVp}$ spectrum. The same regularization parameter $\left(\gamma_{1}=\gamma_{2}=2^{-5}\right)$ for soft tissue and bone was used for PWLS and PL. For fair comparisons, we applied a spatial kernel given by $K(r) \triangleq\{0.25,0.5,0.25\}$ for smoothing in the radial direction to the restored component sinogram by the conventional decomposition only. We pursue more rigorous analysis to match the spatial resolutions of restored component sinograms by three methods elsewhere.

We compared the performance of the conventional sinogram decomposition, the sinogram decomposition by PWLS, and the sinogram restoration by PL in the sinogram domain and in the image domain. Table 1 shows their normalized root mean squared (NRMS) errors. According to Table 1, the PL approach provides the best results among three methods and PWLS is better than the conventional sinogram decomposition in terms of NRMS errors in the restored sinograms and reconstructed images. Fig.2 shows the true component sinograms (soft tissue and bone) and the restored component sinograms by three methods. The reconstructed images by FBP are shown on Fig.3. We conclude that the PWLS and PL approaches provide less noisy restored component sinograms and reconstructed images than the conventional method from Fig.2 and Fig.3. These figures support the results in the top four rows of Table 1.

Since restored component sinograms can be used for attenuation correction in PET images directly, another way to compare the three methods is in terms of ACF defined as follows: For $i=1, \ldots, N_{d}$,

$$
\left.\phi_{i} \triangleq \exp \left(\sum_{l=1}^{L_{0}} \beta_{l}(\mathcal{E}) \widehat{\boldsymbol{s}}_{l i}\right)\right|_{\mathcal{E}=511 \mathrm{keV}},
$$




\begin{tabular}{|c||c|c|c|}
\hline \multicolumn{1}{|c||}{} & \multicolumn{3}{c|}{ Sinogram restoration method } \\
\hline NRMS error & Conventional decomposition & PWLS decomposition & PL restoration \\
\hline \hline Sinogram of soft tissue & $21 \%$ & $13 \%$ & $12 \%$ \\
Sinogram of bone & $56 \%$ & $34 \%$ & $30 \%$ \\
\hline Image of soft tissue & $54 \%$ & $33 \%$ & $31 \%$ \\
Image of bone & $64 \%$ & $42 \%$ & $41 \%$ \\
\hline Attenuation correction factors & $22 \%$ & $9 \%$ & $8 \%$ \\
\hline
\end{tabular}

Table 1. The NRMS errors of restored component sinograms (top two rows), reconstructed component images (middle two rows), and attenuation correction factors (ACF) (bottom row) by the conventional decomposition, PWLS decomposition, and PL restoration. The image reconstructions are performed by FBP.

where $\widehat{\boldsymbol{s}}_{l i}$ denotes the restored component sinograms for the $l$ th material type. The ACFs are used to correct the attenuation in PET images by compensating the survival probability. The bottom row of Table 1 shows the NRMS errors of ACFs. We conclude that the proposed statistical approaches outperform the conventional sinogram decomposition in terms of NRMS errors.

\section{CONCLUSIONS}

We proposed two statistical sinogram restoration methods in low-dose DECT for attenuation correction of PET images. In the simulations, the proposed PL provided the lowest NRMS errors among three approaches and PWLS yielded much lower NRMS errors than the conventional decomposition in the sinogram domain, in the image domain, and in terms of ACFs. These methods showed promise for PET attenuation correction from low-dose DECT sacns.

\section{ACKNOWLEDGEMENTS}

This research is supported in part by NIH 1R01CA115870.

\section{REFERENCES}

1. P. E. Kinahan, D. W. Townsend, T. Beyer, and D. Sashin, "Attenuation correction for a combined 3d PET/CT scannter," Med. Phys. 25, pp. 2046-53, Oct. 1998.

2. P. E. Kinahan, B. Hasagawa, and T. Beyer, "X-ray-based attenuation correction for positron emission tomography / computed tomography scanners," Sem. Nuc. Med. xxxiii, pp. 166-79, July 2003.

3. J. A. Fessler, I. Elbakri, P. Sukovic, and N. H. Clinthorne, "Maximum-likelihood dual-energy tomographic image reconstruction," in Proc. SPIE 4684, Medical Imaging 2002: Image Proc., 1, pp. 38-49, 2002.

4. P. Kinahan, J. A. Fessler, A. Alessio, and T. K. Lewellen, "Quantitative attenuation correction for PET/CT using iterative reconstruction of low-dose dual-energy CT," in Proc. IEEE Nuc. Sci. Symp. Med. Im. Conf., 5, pp. 3285-9, 2004.

5. P. E. Kinahan, A. M. Alessio, and J. A. Fessler, "Dual energy CT attenuation correction methods for quantitative assessment of response to cancer therapy with PET/CT imaging," Technology in Cancer Research and Treatment 5, pp. 319-28, Aug. 2006.

6. P. J. La Riviere, J. Bian, and P. A. Vargas, "Penalized-likelihood sinogram restoration for computed tomography," IEEE Trans. Med. Imag. 25, pp. 1022-36, Aug. 2006.

7. N. H. Clinthorne, "A constrained dual-energy reconstruction method for material-selective transmission tomography," Nucl. Instr. Meth. Phys. Res. A. 353, pp. 347-8, Dec. 1994.

8. R. E. Alvarez and A. Macovski, "Energy-selective reconstructions in X-ray computed tomography," Phys. Med. Biol. 21, pp. 733-44, Sept. 1976.

9. J. Hsieh, "Adaptive streak artifact reduction in computed tomography resulting from excessive x-ray photon noise," Med. Phys. 25, pp. 2139-47, Nov. 1998.

10. J. A. Fessler, "Hybrid Poisson/polynomial objective functions for tomographic image reconstruction from transmission scans," IEEE Trans. Im. Proc. 4, pp. 1439-50, Oct. 1995. 

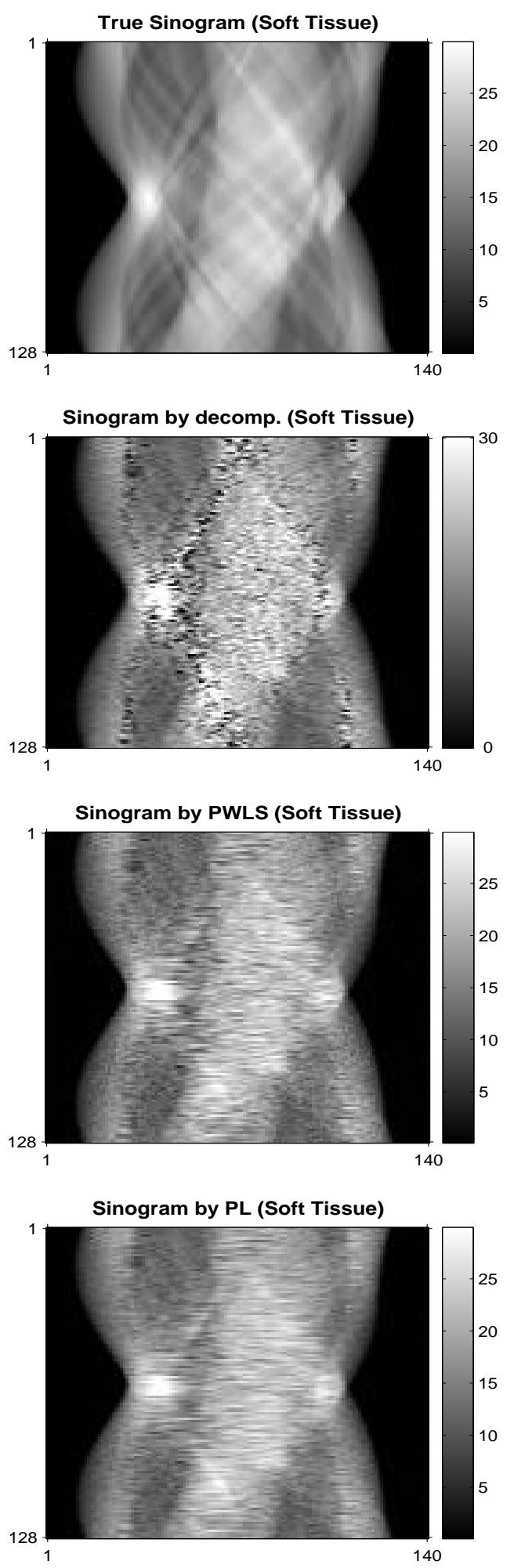
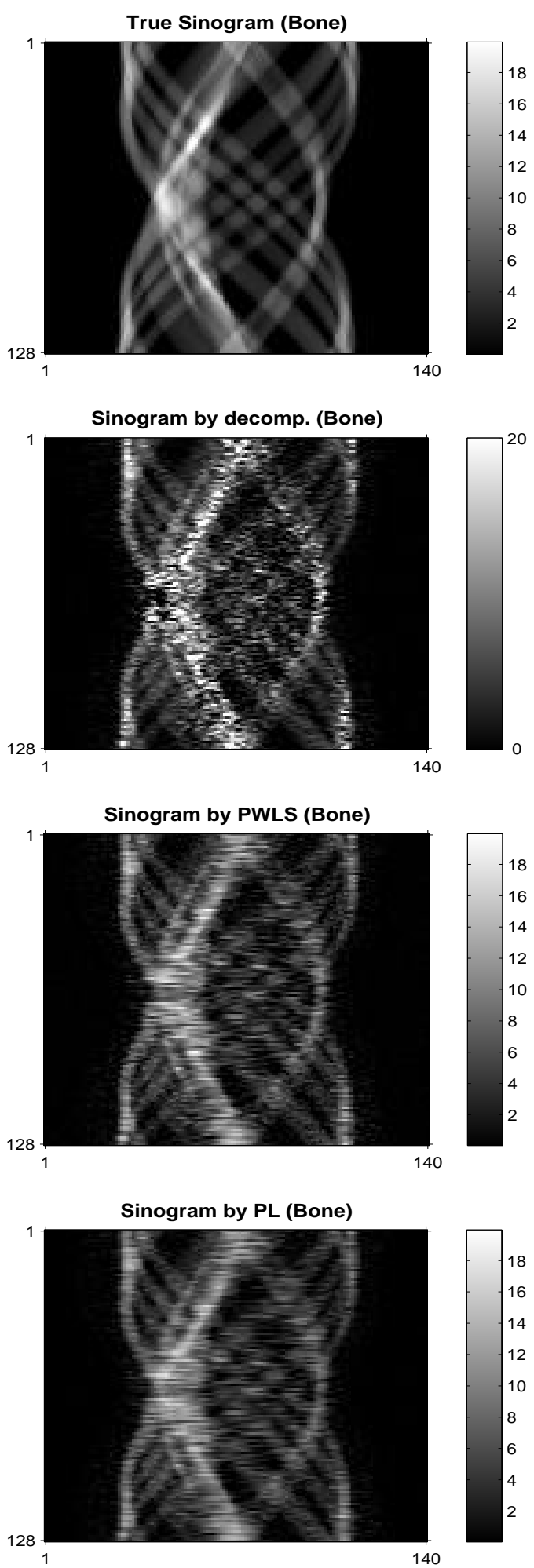

Figure 2. The restored component sinograms (soft tissue and bone) by the conventional decomposition (the 2nd row), PWLS decomposition (the 3rd row), and PL restoration (the 4th row) with true sinograms (the 1st row). 

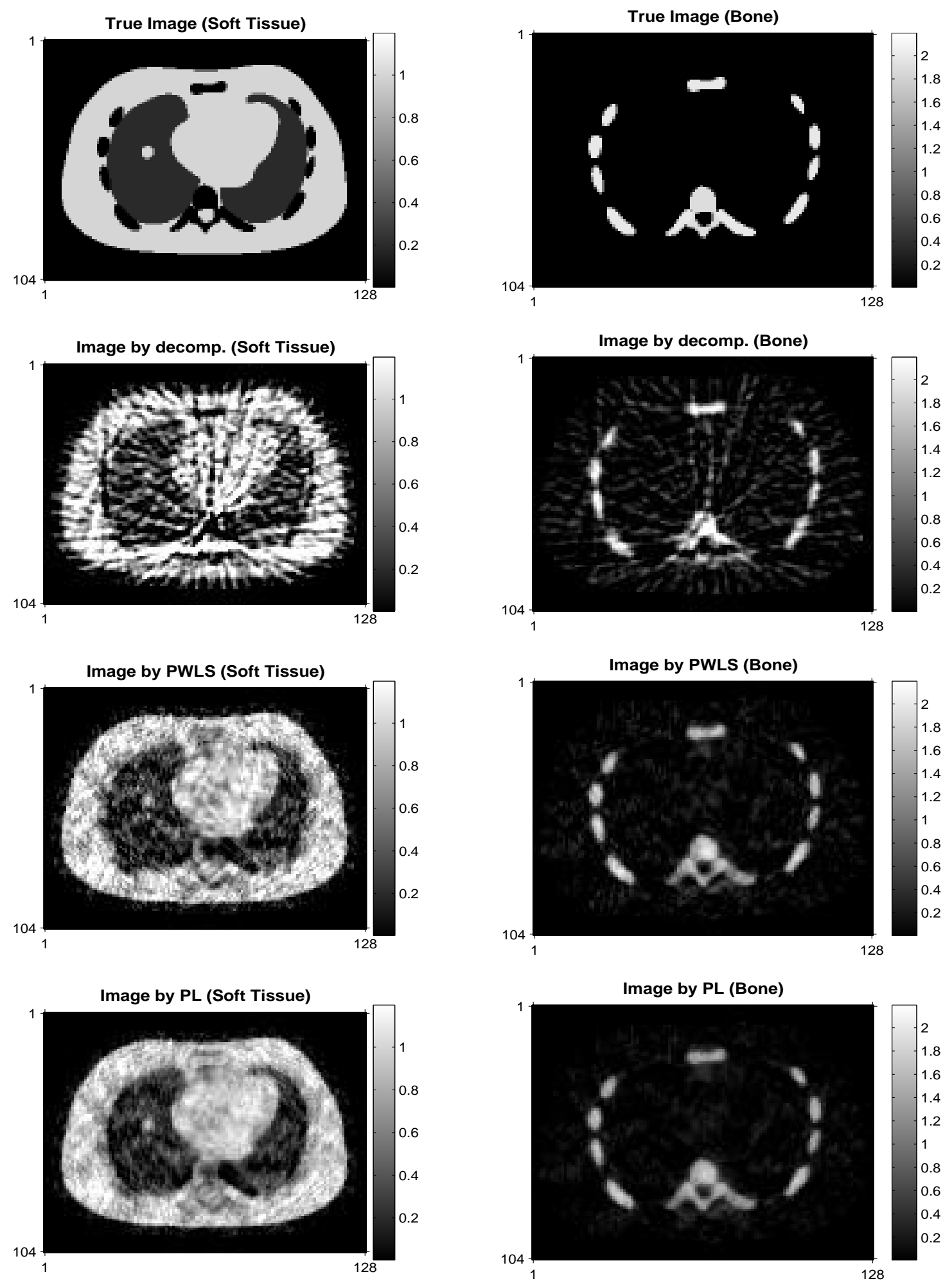

Figure 3. The reconstructed component images (soft tissue and bone) by the conventional decomposition (the 2nd row), PWLS decomposition (the 3rd row), and PL restoration (the 4th row). Applying FBP to the component sinograms in Fig.2 yields these images. The true density maps of soft tissue (left) and bone (right) are shown in the 1st row. 
11. J. A. Fessler, "Mean and variance of implicitly defined biased estimators (such as penalized maximum likelihood): Applications to tomography," IEEE Trans. Im. Proc. 5, pp. 493-506, Mar. 1996.

12. M. W. Jacobson and J. A. Fessler, "An expanded theoretical treatment of iteration-dependent majorizeminimize algorithms," IEEE Trans. Im. Proc. 16, pp. 2411-2422, Oct. 2007. 